\title{
Systems biology: unlocking the complexities of disease to enhance medicine
}

\author{
“...trends in changes of levels versus absolute values of specific biomarkers ... may \\ better reflect the progression of pathology compared with a cross-sectional single \\ time point measurement."
}

\begin{abstract}
Keywords: biomarker $\bullet$ longitudinal study $\bullet$ personalized medicine $\bullet$ systems biology approach
\end{abstract}

The current emphasis on clinical symptom management and treatment, rather than preventative care, leads to systemic inefficiencies and is a key contributing factor to the rising costs of healthcare. The lack of early detection and effective treatment for many ailments also contributes to this problem. Despite significant amounts of resources, one of the reasons for high attrition rates in drug discovery processes is the focus on specific therapeutic targets instead of viewing disease as a whole [1]. Recent disappointing results from several companies targeting aggregated amyloid protein for Alzheimer's disease treatment illustrates the deficiencies of single-target-based drug discovery processes [2]. Once a promising drug candidate is identified, the protocol that is adapted in clinical development reveals further flaws. The current design of clinical trials evaluates therapeutic efficacy within a group of participants, but beneficial effects can easily be lost due to numerous intrinsic (genetic) and extrinsic (environmental) factors between participants that are outside of researcher's control [3]. Focusing on therapeutic responses from stratified patient populations based on specific indicators may increase the success rate in clinical development, provide new therapeutic leads for subsets of patients or patients with specific pathologies and fuel the development of personalized medicine. However, this approach is hindered by the lack of appropriate biomarkers for the vast majority of complex diseases.

\section{Systems biology}

Being able to comprehensively assess molecular changes in order to investigate the underlying processes involved in the etiology and progression of diseases has long been a dream of researchers. The development and adoption of various high-throughput measurement technologies for the genome, transcriptome, proteome, metabolome and others have completely shifted the bottleneck from data generation to data integration/interpretation. This also leads to the infusion of new disciplines, especially computation, statistics and mathematical modeling, into biological research. The advances in technologies looking at global molecular changes at different levels leads to the possibility of exploring what processes, pathways or networks are associated with a specific biological state. This drives the development of an integrated approach towards a holistic view of biology - the 'systems biology'.

Despite there being different definitions of systems biology, the core of this approach contains two integral parts: the capability of conducting accurate global measurements at different levels; and the ability to integrate different datasets in order to build molecular networks/models [4]. This systematic approach can generally be divided into several key components: conduct accurate global measurements of biomolecules at different levels (from the genome to the epigenome and metagenome); capture and integrate information from different levels in order to build biological networks; model the responses of biological networks to different perturbations; and test and refine the models with additional experimental datasets. In order to take the full advantage of this approach, longitudinal sampling is critical, since it can capture dynamic

\section{Yuqing $\mathrm{He}$}

Institute of Medical Systems Biology, Guangdong Medical College, Dongguan, Guangdong 523808, China

\section{Kathie-Anne Walters}

Institute for Systems Biology, Seattle, WA 98109, USA

Inyoul Lee

Institute for Systems Biology, Seattle, WA 98109, USA

\section{Kai Wang}

Author for correspondence: Institute for Systems Biology, Seattle, WA 98109, USA

kwang@systemsbiology.org 
changes in networks so that key molecules that are responsible for these changes can be identified.

\section{Systems biology \& complex disease}

Systems biology approaches can shed light on the biological networks that are perturbed in diseases, such as the identification of the biological processes involved in the pathogenesis of prion disease [5] and the comprehensive characterization of various cancer types through The Cancer Genome Atlas project [6]. These findings can subsequently be used in order to assess how well various therapies maintain or restore normal biological processes, ultimately facilitating the development of more effective therapeutics or preventative measures based on network responses as opposed to individual therapeutic targets. The systems approach has also been used in order to understand both the beneficial and adverse effects of drugs. A recent analysis of a publicly available database - the US FDA's Adverse Event Reporting System - helped researchers to understand the drug-target interactions that may lead to adverse phenotypes [7]. This study further demonstrated the possibility of identifying drug combinations with reduced adverse effects by understanding the interactions of therapeutic targets within the context of cellular regulatory networks.

\section{"...sampling is critical, since it can capture dynamic changes in networks so that key molecules that are responsible for these changes can be identified."}

Identifying proper control samples is critical for systems biology approaches. Currently, the most common method is to compare diseased samples with adjacent normal samples from patients or, if possible, match normal tissue samples from healthy volunteers. However, adjacent tissue may not be completely normal, especially in the case of cancers. Genetic differences and environmental factors may make identifying samples form matched volunteers impossible. Besides the well-characterized clinical samples, the success of systems biology also depends on suitable in vitro or in vivo model systems in order to depict the disease of interest, such as the use of animal models in order to understand the pathogenesis of glioblastoma multiforme and the cardiovascular ailments associated with post-traumatic stress disorder [8,9]. Combining results from well-characterized in vitro or in vivo models with human patient samples is a powerful approach since it offers the opportunity for the precise manipulation of specific biological networks in order to test their disease association and capture dynamic changes. However, it is important to recognize the deficiencies of models: most models can only focus on specific aspects of a disease. In vivo models rarely reflect the genetic complexity inherent in human populations, which plays an important role in the majority of diseases. In addition, in vitro models lack the complex cellular environments that drive the development of pathology.

\section{Systems biology \& personalized medicine}

Since global measurement tools have become more affordable, it is now possible to conduct repeated comprehensive measurements from an individual. These types of longitudinal data are extremely useful for identifying and monitoring the changes of perturbed networks under defined conditions (genetic and environmental) prior to and during the onset of disease development. The well-known study from Smarr who conducted a longitudinal study on himself provides significant insights into the power of this approach [10]. A number of initiatives, including the Hundred Person Wellness pilot project, are testing the feasibility of using systems approaches in healthy individuals in order to develop personalized, preventative measures based on the results of longitudinal omics data [11]. Repetitive measurements also provide the opportunity to use a different type of indicator: trends in changes of levels versus absolute values of specific biomarkers. This type of information may be more accurate and informative since even minor changes in repetitive measurements may better reflect the progression of pathology compared with a cross-sectional single time point measurement. In addition, this approach reduces the impact that various intrinsic and extrinsic factors, including biological and measurement noise, may have on the levels of specific biomarkers.

\section{Challenges}

Systems biology provides a path towards integrating and extracting meaningful biological information from various datasets in order to gain insights into complex biology and disease. However, there are a number of critical issues that may impact the success of this approach. These include the ability to: develop in vitro and in vivo model systems that accurately reflect a specific biological state of interest; identify informative biomarkers for the disease of interest; use proper and standardized protocols for sample procurement and preparation; conduct repetitive and accurate measurements in order to acquire different levels of biological information; and develop suitable computation tools in order to extract and integrate different types of data. Even though systems biology emphasizes the integration of global measurements, the foundation of data integration is based on detailed experimental results on individual molecules. In the past, we have only been able to study individual molecules in a system, 
similarly to individual pixels in a picture. Just as precisely placed individual pixels can form a beautiful picture, systems biology allows us to arrange thousands of individual, detailed studies into a holistic view of a complex biological system.

\section{Financial \& competing interests disclosure}

The authors gratefully acknowledge support from DOD research contracts W911NF-10-2-0111 and HDTRA1-13-C-0055, grants from the National Science Foundation of China 81201223 (Y He), the Guangdong Province Medical Research Foundation A2012533 (Y He), the Science and Technology

\section{References}

1 Kell DB. Finding novel pharmaceuticals in the systems biology era using multiple effective drug targets, phenotypic screening and knowledge of transporters: where drug discovery went wrong and how to fix it. FEBS J. 280(23), 5957-5980 (2013).

2 Cedernaes J, Schioth HB, Benedict C. Efficacy of antibodybased therapies to treat Alzheimer's disease: just a matter of timing? Exp. Gerontol. 57, 104-106 (2014).

3 Bielekova B, Vodovotz Y, An G, Hallenbeck J. How implementation of systems biology into clinical trials accelerates understanding of diseases. Front. Neurol. 5, 102 (2014).

4 Ideker T, Galitski T, Hood L. A new approach to decoding life: systems biology. Annu. Rev. Genomics Hum. Genet. 2, 343-372 (2001).

5 Hwang D, Lee IY, Yoo $\mathrm{H}$ et al. A systems approach to prion disease. Mol. Syst. Biol. 5, 252 (2009).
Program of Guangdong 20130B0510000580 (Y He), the International Science and Technology Cooperation Program of Dongguan 201350815200112 (Y He), the Guangdong Medical College Research Startup Fund B2013001 (Y He) and the Guangdong Medical College Integration of Industry, Education and Research C2013004 (Y He). The authors have no other relevant affiliations or financial involvement with any organization or entity with a financial interest in or financial conflict with the subject matter or materials discussed in the manuscript apart from those disclosed.

No writing assistance was utilized in the production of this manuscript.

6 Cancer Genome Atlas Research Network. Comprehensive molecular characterization of urothelial bladder carcinoma. Nature 507(7492), 315-322 (2014).

7 Zhao S, Nishimura T, Chen Y et al. Systems pharmacology of adverse event mitigation by drug combinations. Sci. Transl. Med. 5(206), 206ra140 (2013).

8 Song Y, Zhang Q, Kutlu B et al. Evolutionary etiology of high-grade astrocytomas. Proc. Natl Acad. Sci. USA 110 (44), 17933-17938 (2013).

9 Cho JH, Lee I, Hammamieh R et al. Molecular evidence of stress-induced acute heart injury in a mouse model simulating posttraumatic stress disorder. Proc. Natl Acad. Sci. USA 111(8), 3188-3193 (2014).

10 Smarr L. Quantifying your body: a how-to guide from a systems biology perspective. Biotechnol. J. 7(8), 980-891 (2012).

11 Gibbs WW. Medicine gets up close and personal. Nature 506(7487), 144-145 (2014). 